Minireview

\title{
Panmixia postponed: ancestry-related assortative mating in contemporary human populations
}

\author{
Andrew S Burrell and Todd R Disotell
}

Address: Center for the Study of Human Origins, Department of Anthropology, New York University, 25 Waverly Place, New York, NY 10003, USA.

Correspondence: Todd Disotell. Email: todd.disotell@nyu.edu

\begin{abstract}
A study of two different populations reveals that in both the choice of a spouse is non-random not only in respect of broad ethnic group but also in regard to specific ancestries within that group. The cause of this surprising bias remains unclear.
\end{abstract}

The past 500 years have been characterized by unprecedented episodes of human migration and admixture, particularly in the Americas. Technological innovations have to a certain extent reduced the impact of geography on human behavior, raising the possibility of a truly global population. At a local level, however, geographic, demographic, linguistic, cultural and even legal barriers now, and in the past, limit and circumscribe human mate choices. For example, cultural biases towards patrilocal or matrilocal marriage (where the married couple set up home in the place of origin of the man or woman, respectively) can lead to the differential structuring of male or female genetic variation [1]. Caste systems can similarly lead to the stratification of genetic structure within societies [2]. The patterns of divergence and admixture that characterize human populations are the result of complex cultural and evolutionary processes, but can also negatively influence the outcomes of biomedical studies associating disease susceptibilities and other biomedical traits with particular genes [3].

In this context, a paper by Risch et al. [4] in Genome Biology is especially interesting in that they used 'ancestry informative markers' (AIMs) to document the genetic signature of assortative mating in contemporary human populations. These genetic markers document the contribution of particular ancestral groups to an individual's genetic make-up. Surprisingly, in view of the fact that such ancestral contributions may not be physically obvious or even known to the individual or their intended spouse, Risch et al. find that ancestral make-up is positively correlated with spouse choice within both populations studied, but find no correlation with socioeconomic or geographic origins that might explain the correlation. The work raises interesting questions about the cultural factors influencing human population genomic structure as well as the evolutionary and biomedical significance of such structure.

\section{Ancestral correlations}

Risch et al. [4] examine spousal correlations in genetic ancestry from four Latino populations: Mexicans from Mexico City and the San Francisco Bay Area, and Puerto Ricans from Puerto Rico and New York City. These populations were chosen because of the history of population admixture in the New World, primarily involving three distinct groups: Africans, Europeans and Native Americans. As these 'ancestral populations' are geographically situated at the ends of genetic clines (which display continuous variation in allele frequencies or geographic distance) they are somewhat genetically distinct (Figure 1). Using enough genetic markers, individual genotypes can be compared to the ancestral populations and reasonably assigned a probability of ancestry in each.

To do this the authors used a battery of more than 100 previously defined AIMs that differentiate the three ancestral populations fairly well [5]. These markers, which have no obvious phenotypic correlations, were then used to assess each individual's proportion of ancestry in each of the ancestral populations. Proxies for the ancestral populations came from around 30 samples each of 'west Africans', 'European Americans' from the Coriell Institute, and Mayan and Nahua Native Americans [5]. The AIMs used are biallelic single nucleotide polymorphisms (SNPs) chosen from a SNP chip panel because of high interpopulational allele-frequency differences (at least 0.5) and because they are widely distributed across the genome. Wide genomic distribution minimizes the possibility of linkage disequilibrium (LD) between the SNPs in the ancestral populations. (LD refers to an increased likelihood that particular alleles of two different genes will be inherited together rather than being randomly assorted at each generation.) Thus, the pattern of any LD seen between these loci in the current populations may indicate admixture and/or assortative (non-random) mating.

Spouse pairs were recruited from the ongoing Genetics of Asthma in Latino Americans (GALA) project at the 


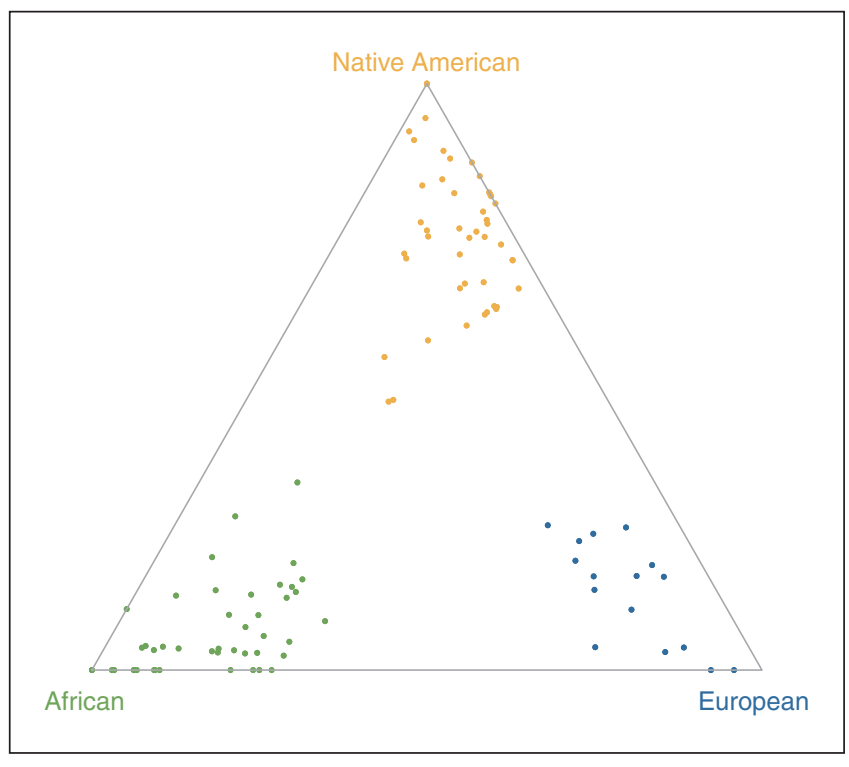

Figure 1

Ancestry-informative markers (AIMs) used to infer ancestry in African, European or Native American populations. Dots represent individual AIMs, and the location of the dot represents its frequency in each ancestral population. AIMs are colored according to the population they uniquely differentiate. AIM frequency data were taken from Table 1 of [5].

University of California, San Francisco, and were self identified as either Mexican or Puerto Rican with all four grandparents also identified likewise. Each individual was genotyped for the full set of AIMs. These genotypes were then used to infer the probable degree of ancestry for each individual in each of the three populations using the program STRUCTURE [6]. STRUCTURE clusters individuals into putative populations in such a way as to maximize Hardy-Weinberg equilibrium and minimize linkage disequilibrium. Individuals are given a probability from 0 to 1 of ancestry in each population, with admixed individuals being defined as those having ancestry in more than one population (Figure 2).

Risch et al. [4] were primarily looking to see whether spouse pairs are correlated in ancestry, and if so, whether socioeconomic status and or geography can account for it. If spouses are correlated in ancestry, it could be a reflection of finding a partner within the same socioeconomic class, which historically may also reflect ethnicity. It is also possible that spouse correlations in ancestry could relate to simple geography, in that spouses are being found in the local community, and local communities could be stratified by ethnicity. Socioeconomic status was inferred from census material or the recruitment center location, while geography was approximated by looking at withinethnicity differences between sites (for example, Mexico City versus Bay Area Mexicans).

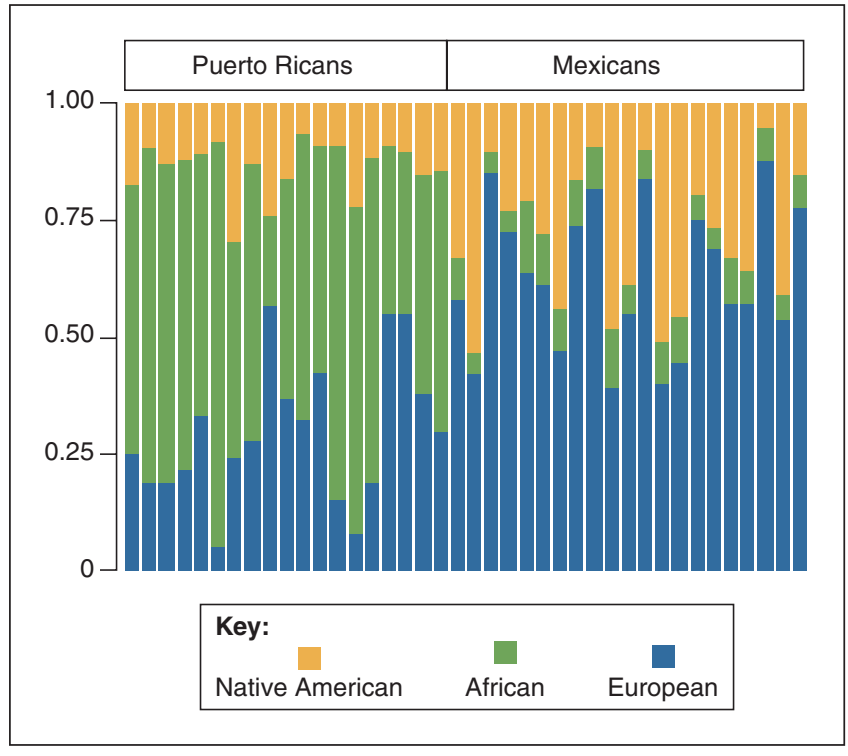

Figure 2

Estimated probability of ancestry in African, European and Native American populations for 20 randomly selected Mexican and Puerto Rican individuals. Ancestry estimates for each person were inferred from Figure 1 of [4].

The analyses yield interesting results. Unsurprisingly, given the history of the two ethnic groups, Mexican ancestry is generally among Native Americans and Europeans, while the Puerto Rican background is of European and African origin. More interestingly, spouse correlations confirm a tendency towards marriage between people with similar ancestry. In Mexicans, correlations between spouses in Native American ancestry and between European ancestry are significant and positive (Figure 3). Likewise, for the Puerto Ricans, spouse correlations in European and African ancestry are significant and positive, although the signal of assortative mating is not as strong as in the Mexican group. Values of the correlation coefficient $r^{2}$ range from 0.57 for European ancestry in Bay Area Mexicans to 0.24 for European ancestry in all Puerto Ricans.

Socioeconomic and geographic correlations are not strong and only weakly explain the patterns of assortative mating with regard to ancestry. Spouse correlations in ancestry continue even within socioeconomic class and within geographic subgroupings. In terms of geography, the only significant difference within ethnic groups is that Mexicans from Mexico City have significantly more Native American ancestry than those from the Bay Area. Puerto Ricans from New York are not significantly different form those from Puerto Rico itself. Estimated socioeconomic effects are likewise small, with the only significant correlation being a positive relationship between African ancestry and Puerto Rican women. 


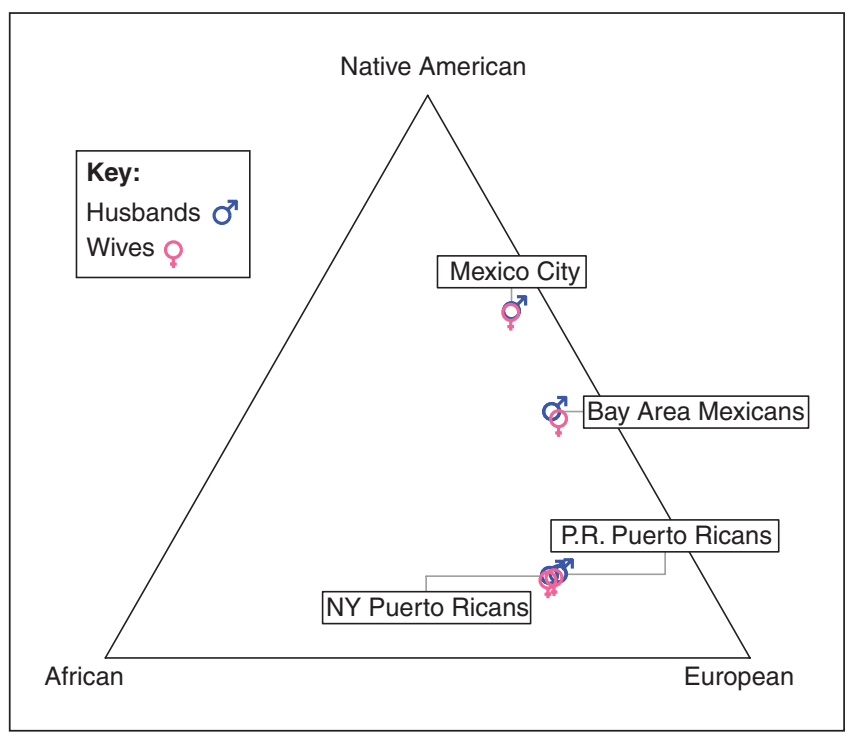

Figure 3

Average group ancestries in African, European and Native American populations stratified by sample locality and sex. Average group ancestry data are from Table 1 of [4]. Note that despite the range of ancestry in Figure 2, spouse pairs are more similar than expected by chance. P.R., Puerto Rica.

Analyses of the genomic structure of the groups studied reveal patterns of admixture consistent with the STRUCTURE results. Non-random mating and/or admixture are likely to produce associations between loci that are not closely linked, and statistical tests did in fact suggest that a relatively high proportion of these loci are in LD. LD is strongest among markers that differentiate Europeans from Native Americans in the Mexican populations, and among markers separating Africans and Europeans in the Puerto Rican populations.

The signal of admixture in these two sample ethnic groups is clear, with both the spousal correlations and the linkage analyses showing different patterns of mixed ancestry in the Mexican and Puerto Rican populations. The spousal correlations also resulted in a relatively clear sign of ancestry-related assortative mating in the two groups. The cause of this assortative mating, however, is less clear. The authors find only slight indications of correlations with socioeconomic status and therefore argue that the correlation in ancestry between spouses is not being driven by a preference for partners from the same economic class. This is perhaps surprising, given that, until recently, ethnicity and socioeconomic class were correlated, at least in the United States $[7,8]$. Nevertheless, the association between spouses in ancestry remains even when looking within classes, and may be reliable.

The authors also dismiss geography as a driver of the ancestry correlation, but here their argument is weaker.
There was a geographic effect in the Mexican study group, with Mexicans from Mexico City having significantly more Native American ancestry than those in the Bay Area. This could reflect admixture with individuals of European descent in the Bay Area, or could reflect a bias in migration to California by Mexicans with more European ancestry. One possible problem confounding the attempt to find a geographic association is that the analyses may simply be too large in geographic scale to produce a signal: if individuals are choosing partners from their local community or neighborhood, and local communities are structured by ethnicity, this effect may not be seen within a larger geographic area.

\section{Culture and human population structure}

The causes of human population structure vary from simple isolation by distance to - as this case suggests human cultural behavior. Basic cultural practices such as patrilocality or matrilocality can have an effect on the structuring of maternally, paternally and biparentally inherited genetic variation $[1,9]$. On a global level, several studies have suggested that Y-chromosomal variation is more geographically localized than autosomal or mitochondrial variation, suggesting a general tendency among societies towards patrilocality and female emigration, with periodic episodes of long-range migration by males (reviewed in [10]). However, recent studies have questioned the strength of Y-chromosomal structuring, and it may be that changes in human cultural customs related to the adoption of agriculture [11] or demographic differences related to cultural practices [12] have further affected current population structure.

Ancestry-related assortative mating in many organisms is related to specific mate recognition, but in humans, withingroup mating is likely to be partly linked to cultural factors. Many countries until recently legally barred men and women of different races from marrying. Since the overturning of such laws in the United States after 1967 by the Supreme Court in Loving vs Virginia, rates of interracial marriage have substantially increased [13]. Census data collected in the United States in the 1990s and corrected for local demographic characteristics display interesting patterns of interracial unions. The group most likely to marry outside of their ethnic group is Asian women (35.9\%). Asian men are far less likely to do so (23\%); conversely, black men are more likely to intermarry with women from other groups (9.8\%) than black women (4.1\%) [14].

These kinds of generational group- and gender-specific patterns seem, at least on the surface, more likely to be the result of cultural rather than biological factors. However, while rates of intermarriage may have increased, most individuals are still overwhelmingly likely to find mates within their group. Demographics also play a role in ancestry-related assortative mating: recent surveys of 
interracial marriage show declining trends among Asians and Latinos, possibly related to an increasing availability of within-group mates as the relative population size of each group within the United States expands [15]. Interestingly, global immigration patterns and population movement may counterintuitively result in greater ancestry-related assortative mating, at least in the short term, by increasing the local pool of mates from a similar ethnic background. Risch et al. [4] persuasively argue that ancestry-related assortative mating is occurring in the Latin American populations. Why it occurs and how it is maintained remains unclear, and is a fertile field for future research.

The presence of current and historical population structure affects studies of human evolution, population history and health. From an evolutionary genetic perspective, ancestryrelated assortative mating will increase the overall human 'effective population size' $\left(N_{\mathrm{e}}\right)$ while simultaneously decreasing it in local populations, thus enhancing differences among groups. From a more practical viewpoint, this type of nonrandom mating will potentially confound association studies by increasing LD. Therefore, the effects of human behaviors like assortative mating need to be addressed in the design and interpretation of future association studies.

\section{Acknowledgements}

We would like to thank C Bergey and J Hodgson for helpful discussions and assistance.

\section{References}

1. Oota $\mathrm{H}$, Settheetham-Ishida $\mathrm{W}$, Tiwawech $\mathrm{D}$, Ishida $\mathrm{T}$, Stoneking M: Human mtDNA and Y-chromosome variation is correlated with matrilocal and patrilocal residence. Nat Genet 2001, 29:20-21.

2. Bamshad M, Kivisild T, Watkins WS, Dixon ME, Ricker CE, Rao BB, Naidu JM, Prasad BV, Reddy PG, Rasanayagam A, Papiha SS, Villems R, Redd AJ, Hammer MF, Nguyen SV, Carroll ML, Batzer MA, Jorde LB: Genetic evidence on the origins of Indian caste populations. Genome Res 2001, 11:994-1004.

3. Lander ES, Schork NJ: Genetic dissection of complex traits. Science 1994, 265:2037-2048.
4. Risch N, Choudry S, Via Garcia M, Basu A, Sebro R, Eng C, Beckman K, ThyneS, Chapela R, Rodriguez-Santana JR, Rodriguez-Ciontron W, Avila PC, Ziv E, Burchard EG: Ancestry-related assortative mating in Latino populations. Genome Biol 2009, 10:R132.

5. Yeager R, Avila-Bront A, Abdul K, Nolan PC, Grann VR, Birchette MG, Choudry S, Burchard EG, Beckman KB Gorroochurn P, Ziv E, Consedine NS, Joe AK: Comparing genetic ancestry and self-described race in AfricanAmericans born in the United States and Africa. Cancer Epidemiol Biomarkers Prev 2008, 17:1329-1338.

6. Pritchard JK, Stevens M, Donnelly P: Inference of population structure using multilocus genotype data. Genetics 2000, 155:945-959.

7. Newburger EC, Curry A: Educational Attainment in the United States: March 1999. US Census Bureau, Current Population Reports, Series P20-528, Washington, DC; 2000.

8. McKinnon J: The Black Population in the United States: March 2002. US Census Bureau, Current Population Reports, Series P20-541, Washington, DC; 2003.

9. Destro-Bisol G, Donati F, Coia V, Boschi I, Verginelli F, Caglia A, Tofanelli S, Spedini G, Capelli C: Variation in female and male lineages in sub-Saharan Africa: the importance of sociocultural factors. Mol Biol Evol 2004, 21:1673-1682.

10. Disotell TR: Human evolution: sex-specific contributions to genome variation. Curr Biol 1999, 9:R29-R31.

11. Wilkins JF, Marlowe FW: Sex-biased migration in humans: what should we expect from genetic data? BioEssays 2006 , 28:290-300.

12. Ségurel L, Martínez-Cruz B, Quintana-Murci L, Balaresque $P$, Georges M, Hegay T, Aldashev A, Nazyrova F, Jobling MA Heyer E, Vitalis R: Sex-specific genetic structure and social organization in Central Asia: insights from a multi-locus study. PLoS Genet 2008, 4:e1000200.

13. Rosenfeld MJ: Racial, educational, and religious endogamy in the United States: a comparative historical perspective. Social Forces 2008, 87:1-31.

14. Harris $\mathrm{DR}$, Ono $\mathrm{H}$ : How many interracial marriages would there be if all groups were of equal size in all places? A new look at national estimates of interracial marriage. Social Sci Res 2005, 34:236-251.

15. Qian Z, Lichter DT: Social boundaries and marital assimilation: interpreting trends in racial and ethnic intermarriage. Am Sociolog Rev 2007, 72:68-94.

Published: 27 November 2009

doi:10.1186/gb-2009-10-11-245

(C) 2009 BioMed Central Ltd 